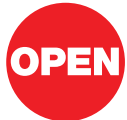

SUBJECT AREAS: NANOTECHNOLOGY IN CANCER

BIOLOGICAL PHYSICS

FLUID DYNAMICS

RADIOTHERAPY

Received

11 January 2013

Accepted

28 January 2013

Published

15 February 2013

Correspondence and requests for materials should be addressed to

E.S. (surdutov@ oakland.edu)

\title{
Biodamage via shock waves initiated by irradiation with ions
}

\author{
Eugene Surdutovich ${ }^{1,2}$, Alexander V. Yakubovich ${ }^{1,3}$ \& Andrey V. Solov'yov ${ }^{1,3}$ \\ ${ }^{1}$ Frankfurt Institute for Advanced Studies, Ruth-Moufang-Str. 1, 60438 Frankfurt am Main, Germany, ${ }^{2}$ Department of Physics, \\ Oakland University, 2200 N. Squirrel Rd., Rochester, Michigan 48309, USA, ${ }^{3}$ A.F. loffe Physical-Technical Institute, \\ Politechnicheskaya 26, 194021 St. Petersburg, Russia.
}

Radiation damage following the ionising radiation of tissue has different scenarios and mechanisms depending on the projectiles or radiation modality. We investigate the radiation damage effects due to shock waves produced by ions. We analyse the strength of the shock wave capable of directly producing DNA strand breaks and, depending on the ion's linear energy transfer, estimate the radius from the ion's path, within which DNA damage by the shock wave mechanism is dominant. At much smaller values of linear energy transfer, the shock waves turn out to be instrumental in propagating reactive species formed close to the ion's path to large distances, successfully competing with diffusion.

. he physics and chemistry of radiation damage caused by irradiation with heavy ions has recently become a subject of an intense interest because of the use of ion beams in cancer therapy (IBCT) ${ }^{1-3}$. IBCT allows a delivery of high doses into tumors, maximising cancer cell destruction, and simultaneously minimising the radiation damage to surrounding healthy tissue. Ion beam techniques utilise the fundamental difference in energy deposition profile between massive projectiles and massless photons.

The key feature utilised by IBCT is the Bragg peak, which is a sharp maximum in the linear energy transfer (LET) of ions at the end of their trajectories. The Bragg peak occurs as a result of the increase up to the maximum values of the inelastic cross sections of interactions with the molecules of the medium as the speed of the projectile decreases. Therefore, ions deposit most of their destructive energy to the tissue within $1 \mathrm{~mm}$ and the dose deposition quickly drops to almost zero distal to the peak. The location of the Bragg peak depends on the initial energy of ions. Typical depths for carbon ions (in liquid water representing tissue), widely used in IBCT, range from about 8 to $28 \mathrm{~cm}$ as the initial energy ranges from 200 to $430 \mathrm{MeV} /$ nucleon. Hence, a deeply-seated tumour can be scanned with a well focused pencil beam of ions with minimal lateral scattering.

Over the past 20 years, technological and clinical advances of IBCT have developed more rapidly than the understanding of radiation damage with ions. Although an empirical approach has produced exciting results for thousands of patients thus far, many questions concerning the mechanisms involved in radiation damage with ions remain open and the fundamental quantitative scientific knowledge of the involved physical, chemical, and biological effects is, to a significant extent, missing. Indeed, the series of works that elucidated the importance of low-energy (below ionisation threshold) electrons appeared in ca 2000, while the treatment of patients at GSI (Gesellschaft für Schwerionenforschung, Darmstadt, Germany) started in 1997. The molecular mechanism of a double strand break (DSB), the most important DNA lesion, still remains unknown. Even the significance of the relation of DNA damage (including DSBs) compared to the damage of other cellular components to the cell death is not entirely clear. This list can be continued. Besides IBCT, the mechanisms of biodamage due to irradiation with heavy ions have attracted attention in relation with radioprotection from galactic cosmic rays ${ }^{3}$.

The multiscale approach to the physics of radiation damage has the goal of developing this knowledge at the nanoscale and molecular level and finding the relation between the characteristics of incident particles and resultant biological damage ${ }^{1,4}$. This approach is unique in distinguishing phenomena relevant to radiation damage at a given time, space, or energy scale and assessing the resultant damage based on these effects. The significance of understanding the fundamental mechanisms of radiation damage in order to exploit this knowledge for practical applications has inspired the European COST Action ${ }^{5,6}$, which supports collaborations of physicists, chemists, and biologists, studying these phenomena both theoretically and experimentally.

While the Bragg peak location answers a question of where most of the damage occurs, we are raising a question of how the damage happens. Presently, we investigate the effects that stem from a large inhomogeneity of the dose distribution in the vicinity of the Bragg peak on biological damage. They are the consequences of the following scenario. 
The absorbtion of energy by the medium associated with dose results in ionisation and excitation of molecules of this medium. These events happen within $10^{-16} \mathrm{~s}$ after an ion's passage. The next stage of local energy relaxation happens after $10^{-15}$ to $10^{-13} \mathrm{~s}$ when locally produced secondary electrons interact with the medium (effects similar to electron-phonon coupling take place), and the energy becomes distributed between the vibrational and translational degrees of freedom of the molecules. This, according to the predictions of Refs. 7,8 for carbon ions, happens within a cylinder of about one $\mathrm{nm}$ radius, which is so small that the temperature within this cylinder increases by over $1000 \mathrm{~K}^{8}$ by $10^{-13} \mathrm{~s}$ (we will refer to it as the "hot cylinder"). This increase of temperature brings about a rapid increase of pressure (up to $1 \mathrm{GPa}$ ) compared to the atmospheric pressure outside the cylinder. Such circumstances cause the onset of a cylindrical shock wave described by the strong explosion scenario $^{9}$. The pressure rapidly increases on the wave front and then decreases in the wake. The shock wave propagates for about $10 \mathrm{ps,}$ loses energy, and decays. The intriguing questions that were raised in Refs. 8,9 are what such a shock wave can do with biomolecules such as DNA located in the region of its propagation through the medium and what other effects can be explained by the transport related to the shock wave. The forces acting on DNA segments were predicted to be as large as $2 \mathrm{nN}$, which is more than enough to break a covalent bond, causing a strand break; however, these forces are only acting for a short time and it remained unclear whether this is sufficient to cause severe damage to DNA molecules.

\section{Results}

In this work, we study the effects of the interaction of the shock wave formed in a liquid water medium following the traverse of an ion through this medium at different values of LET. Several factors contribute to the values of LET: the charge of the ion, closeness to the Bragg peak, and the composition of the medium. The value of $0.9 \mathrm{keV} / \mathrm{nm}$ corresponds to the Bragg peak of a carbon ion, whose effective charge in that region is about $+3^{10}$. This effective charge is determined by the effect of charge transfer, since ions pick off electrons as they slow down. In general, the LET is proportional to the square of the effective charge of the ion. Therefore heavier ions are characterised with higher values of LET. The actual value of charge fluctuates about the effective value, therefore LET fluctuates as well. For instance, fluctuations of the charge of a carbon ion can change the LET by up to $80 \%{ }^{11}$. In a more dense medium than liquid water, such as tissue that contains sugars, amino acids, and other species, the value of LET may be higher by up to $20 \%{ }^{12}$. As a result the LET for carbon ions (at some segments of their trajectories) in tissue may be as large as $2 \mathrm{keV} / \mathrm{nm}$.

We use the value of LET as a parameter describing the energy propagated by the shock wave and analyse the physical conditions in which its action is significant or even dominant for radiation damage assessment. The first effect of a shock wave is the direct thermomechanical damage of a DNA molecule as a result of interaction with the shock wave, and we explore it using molecular dynamics (MD) simulations. The second effect is the propagation of reacting species as a result of collective motion initiated by the shock wave.

In eucaryotic cells, DNA molecules are packed into chromatin fibers. A nucleosome, a histone-protein octamer wrapped about with a DNA double helix, is the primary structural unit of chromatin. Therefore, we carried out the MD simulations of the interaction of the cylindrical shock wave originating from ion's path with a fragment of a DNA molecule situated on the surface of a nucleosome. This arrangement is shown in Fig. 1; the ion's path is perpendicular to the paper plane as well as the axis of the nucleosome disk. Since the action of the shock wave depends on the distance from the ion's path, we thoroughly study the effect on three base pairs, located between 1.5 and $2.2 \mathrm{~nm}$ from the ion's path. The simulations were done for

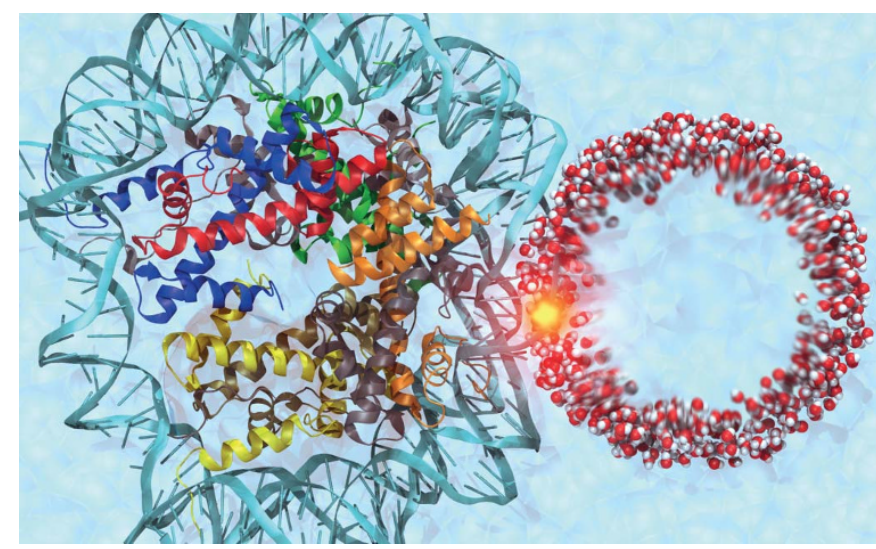

Figure $1 \mid$ (Artistic view) The cylindrical shock wave front in water (on the right; ion's path is the axis of this cylinder, perpendicular to the figure plane) interacts with a nucleosome (on the left) with a segment of a DNA molecule on the surface. The yellow dot indicates the place where damage occurs. The medium is very dense following the wave front and is rarefied in the wake.

four values of LET, 0.9, 1.73, 4.745, and $7.195 \mathrm{keV} / \mathrm{nm}$, corresponding to the predicted values of LET at the Bragg peak for carbon, neon, argon, and iron ions, respectively ${ }^{11}$. Among these, carbon ions are currently the most used ions for heavy-ion therapy. Iron ions are important for the space-mission safety assessment. Between these are neon and argon ions, which are considered for medical applications; they are used in a number of experimental studies ${ }^{3}$.

The radius of the hot cylinder for LET $=0.9 \mathrm{keV} / \mathrm{nm}$ is taken to be $1 \mathrm{~nm}$. This corresponds to the average radius within which secondary electrons lose most of their energy ${ }^{13}$. This estimate can be obtained from the random walk (diffusion) of secondary electrons from the ion's path that gives $l \sqrt{\pi k / 6}$, where $l=0.36 \mathrm{~nm}$ is the elastic mean free path of $35-\mathrm{eV}$ electrons and $k \approx 20$ is the number of collisions after which these electrons lose most of their energy (see the supplemental material).

Noticeable distortion due to the shock wave is apparent 10 ps after the expansion starts. This can be seen in the movie, which can be found in the supplemental material. This distortion comprises the rupture of the secondary structure of the most exposed parts of the DNA molecule, manifested by the nonnative orientation of DNA nucleotides. Many hydrogen bonds are broken, and the bases are located outside the DNA double helix. However, these distortions are reversible, while our main interest is in the investigation of more permanent covalent bond breaking events. Until the computer code allowing us to model bond breaking events is implemented within MBN Explorer software ${ }^{14}$, the most suitable package for the simulation of a shock wave interacting with a nucleosome has been NAMD ${ }^{15}$ with the CHARMM $22^{16}$ force field. In the latter, the interaction potentials used for the description of covalent bonds (relevant for strand breaks) are harmonic. Such a force field does not allow us to observe bond breaking events directly, see description of methods below for further details.

Therefore, in order to study whether the covalent bonds in the DNA backbone can be broken during the shock wave action, we calculated the energy temporarily deposited to these bonds. If this energy exceeded the binding energy of a given bond, we assumed that thermomechanical stresses in the DNA fragment were sufficiently high to break the bond. The corresponding binding energies are referred to as thresholds for breaking the DNA backbone covalent bonds; they are between 3 and $6 \mathrm{eV}^{17}$. Even though the thresholds may be lower (even as low as $0.3 \mathrm{eV}$ ) in the environment consequent the ion's passage ${ }^{18}$, we keep high thresholds in order to obtain conservative estimates for the action of the shock wave. 
The results of the analysis of MD simulations performed for four values of $\operatorname{LET}(0.9,1.73,4.745$, and $7.195 \mathrm{keV} / \mathrm{nm})$ are presented in Fig. 2. The shock wave propagating through a nucleosome transfers part of its energy to covalent bonds of the DNA backbone. Due to the oscillatory motion of the bound atoms the potential energy of the bond oscillates with the characteristic period of $\sim 10 \mathrm{fs}$. Below, we refer to the local maxima in this dependence as the energies carried by the bond during half a period of the oscillation. For the chosen fragment of the DNA backbone located between 1.5 and $2.2 \mathrm{~nm}$ from the ion's path ( 36 bonds) one can calculate the distribution of these bond energies. In MD simulations we have monitored the dynamics of the selected bonds during the time of $10 \mathrm{ps}$, sufficient for the shock wave front to reach the distances $\sim 10 \mathrm{~nm}$, which is well beyond the selected DNA backbone region. During this time the bonds from the selected DNA region make $\sim 1.8 \times 10^{4}$ oscillations. This gives us the total number of bond energy records equal to $N_{r} \approx 3.6 \times 10^{4}$. For further details to this calculation see section Methods below.

The distributions of the bond energy records can be represented by a histogram that assigns to every interval of energy $(E, E+\delta E)$, the number of records corresponding to the bond energies from this interval. For each value of LET used in the simulations, we build such a histogram and refer to it as the bond energies distribution. These distributions (normalised to the total number of records $N_{r}$ for each value of LET) are shown in Fig. 2, where $\ln \left(1 / N_{r} d N / d E\right)$ is plotted vs. corresponding energy interval.

At small values of $E \leq 0.1 \mathrm{eV}$, the distributions for all values of LET coincide, which means that at the considered LETs small excitation energy channels are saturated, i.e., the passing shock wave excites almost every exposed bond, at least somewhat. At higher values of $E$ the distributions deviate from each other and indicate different linear-like dependencies on $E$ for different LETs. This behaviour suggests that the distributions correspond to Boltzmann distributions with different temperatures. The spread of values of $\ln (1 /$ $N_{r} d N / d E$ ) shows that the distributions are not entirely thermalised. They deviate from linear patterns, especially at large energies. Nonetheless, the dominating Boltzmann-like behaviour of these distributions, allows us to fit them as

$$
\frac{1}{N_{0}} \frac{d N}{d E}=\frac{1}{N_{0}} \frac{\delta N}{\delta E}=\frac{1}{k_{B} T} \exp \left[-\frac{E}{k_{B} T}\right],
$$

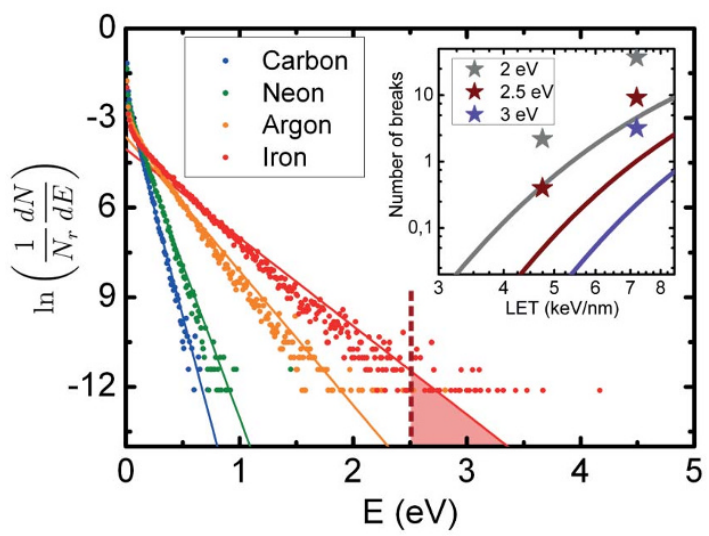

Figure 2 The dependence of logarithm of the normalised number of the covalent bond energy records for the selected DNA backbone region per $0.01 \mathrm{eV}$ energy interval on the bond energy for four values of LET: 0.9, $1.73,4.745$, and $7.195 \mathrm{keV} / \mathrm{nm}$, corresponding to the Bragg peak values for ions of carbon, neon, argon, and iron, respectively. Straight lines correspond to the fits of these distributions. In the inset, the lines correspond to the integrated number of records with energy higher than three thresholds: 2.0, 2.5, and 3.0 eV, calculated using Eq. (4). The stars correspond to exact counts of these records for the four values of LET and corresponding thresholds. where $\delta E=0.01 \mathrm{eV}$ is the width of the energy bin, $\delta N$ is the number of records with energy between $E$ and $E+\delta E$, deposited in selected covalent bonds, normalisation constant $N_{0}$ and temperature $T$ are parameters, and $k_{B}$ is the Boltzmann constant.

Both parameters, $N_{0}$ and $T$, are determined from the fitting of the distributions obtained from MD simulations. Note that the value of parameter $N_{0}=2.17 \times 10^{4}$ is smaller than $N_{r}$ because it corresponds only to the records of relatively large bond energies created by the shock wave propagation. This reduction can be characterised by a dimensionless parameter $a_{0}$, introduced as follows:

$$
a_{0}=\ln \left[\left.\frac{1}{N_{r}} \frac{\delta N}{\delta E}\right|_{E=0}\right]-\ln \left[\left.\frac{1}{N_{0}} \frac{\delta N}{\delta E}\right|_{E=0}\right] .
$$

From fitting, we have derived $a_{0}=-0.52$. Then, from Eq. (2) one obtains $N_{0}=N_{r} \exp \left(a_{0}\right)$. This constant depends on properties of the system being investigated, such as the spatial position of DNA covalent bonds, distance from the ion's path, etc.

Parameters $T$ are the temperatures corresponding to the thermal parts of distributions (for the four values of LET) shown as linear fits in Fig. 2. The values of $T$ are $870,1130,2580$, and $3970 \mathrm{~K}$, indicating that the temperature increase above the temperature of the medium before the interaction with the shock wave, $T_{0}=310 \mathrm{~K}$ (corresponding to a biological system), is directly proportional to LET,

$$
T-T_{0}=\alpha \mathrm{LET},
$$

where $\alpha=494 \mathrm{~K} \cdot \mathrm{nm} \cdot \mathrm{keV}^{-1}$.

The next step is to count the number of energy records of selected covalent bonds of the backbone of a DNA molecule exceeding a given threshold. This is done by direct counting of bond energy records, for which $E>E_{0}$, where $E_{0}$ is a threshold; e.g., for $E_{0}=2.5 \mathrm{eV}$, we count the records to the right of the dashed vertical line in Fig. 2 . The results of such counting, corresponding to the four values of LET and three values of thresholds, are shown as stars in the inset of Fig. 2. [Only five stars, corresponding to $\mathrm{LET}=4.745 \mathrm{keV} / \mathrm{nm}$ for $E_{0}$ equal to 2 and $2.5 \mathrm{eV}$ and to $\mathrm{LET}=7.195 \mathrm{keV} / \mathrm{nm}$ for all three values of $E_{0}$, appear in the range shown.] Alternatively, these numbers can be estimated from integrating Eq. (1) over energies $E$, exceeding a chosen threshold $E_{0}$ :

$$
N=\int_{E_{0}}^{\infty} \frac{d N}{d E} d E=N_{0} \exp \left[-\frac{E_{0}}{k_{B} T}\right] .
$$

This integration corresponds to the area under the curve (shaded triangle in Fig. 2). Since parameters $N_{0}$ and $T$ are fitted, this procedure allows us to predict the number of these over-threshold bond energy records for any value of LET. These numbers correspond to the number of single strand breaks caused by the ion's passage and their dependence on LET is plotted in the inset of Fig. 2. The estimated numbers are smaller than the counted because the estimated numbers rely on the thermal part of the spectrum, while the counting is mostly affected by the non-thermal high-energy "tail" of the spectrum. This along with high values of $E_{0}$ makes our predictions conservative. The inset of Fig. 2 shows that the number of breaks increases very steeply after the critical value of LET is reached; three curves correspond to different thresholds for covalent bond breaking. The number of bond-breaking events, $N$, is equal to the number of strand breaks only until it becomes close to 10 . Larger values of $N$ corresponding to LET $\gtrsim 7 \mathrm{keV} / \mathrm{nm}$ (for $E_{0}=2 \mathrm{eV}$ ) mean that each bond may be broken several times and, therefore, overestimates the number of strand breaks.

In order to compare the number of strand breaks due to the shock wave action to chemical effects, we calculate the probability of a strand break based on bond-breaking events using Poisson statistics. From the predicted number of bond breaking $N$, we can calculate the probabilities for the exact number $v$ of strand breaks to occur, $P(v)=$ 
$\exp (-N) N^{v} / v$ !. Then, the probability $P_{s w}$ of at least one strand break in a given segment of a DNA molecule is equal to $1-P(0)$, i.e.,

$$
P_{s w}=1-\exp (-N) \text {. }
$$

The dependence of this probability on LET for different thresholds is shown in Fig. 3, where we compare this probability to the probability of producing a single strand break owing to chemical effects in a similar DNA segment located at the same distance from the ion's path. The probability of SSBs due to chemical effects, $P_{c h}$, is estimated (see the supplemental material) as

$$
P_{c h}=\beta \frac{\mathrm{LET}}{\mathrm{LET}_{0}},
$$

where $\beta=(1.1 \pm 0.5) \times 10^{-3}$ and $\mathrm{LET}_{0}=0.9 \mathrm{keV} / \mathrm{nm}$. The uncertainty in this expression is shown in Fig. 3 by the shaded area.

\section{Discussion}

From the analysis of Fig. 3, we can infer an important result: for a given threshold, the shock wave breaking effect starts at a certain critical value of LET. After that, the probability increases very steeply, quickly overcoming chemical effects that include interactions of DNA molecules with free radicals, secondary electrons, holes, solvated electrons, etc. Fig. 3 indicates that breaking bonds due to the shock wave mechanism starts (for the $3-\mathrm{eV}$ threshold) at LET $\approx$ $4 \mathrm{keV} / \mathrm{nm}$ and by $5 \mathrm{keV} / \mathrm{nm}$ it becomes the dominant effect in radiation damage. Two smaller thresholds of 2 and $2.5 \mathrm{eV}$ are shown for a comparison.

From this example we can conclude that ions with LET larger than $5 \mathrm{keV} / \mathrm{nm}$, e.g., for ions heavier than $\mathrm{Ar}$ in liquid water, the bond breaking due to the shock wave effect is dominant when DNA segments are within about $2 \mathrm{~nm}$ of the ion's path. The strength of a shock wave is proportional to the pressure at the wave front. In Ref. 9, it is shown that the pressure at the wave front is proportional to the square of the speed of the wave front. This speed is proportional to $\sqrt{\operatorname{LET}} \rho^{-1}$, where $\rho$ is the distance from the ion's path, therefore the pressure and, hence, the forces stretching the bonds, is proportional to LET $\rho^{-2}$ (see the supplemental material). Based on inferences from our simulations, we can estimate the radius of the shock wave effect dominance, $\rho_{\max }$, as

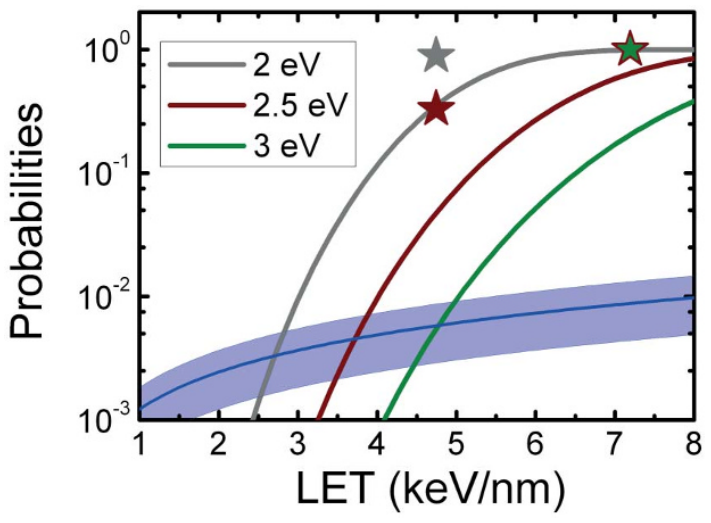

Figure $3 \mid$ The dependence of the probability of producing at least one SSB in a 3-base-pair segment of DNA molecule located between 1.5 and $2.2 \mathrm{~nm}$ from the ion's path on LET: chemical mechanisms (blue line with the uncertainty shadow around it) vs. shock wave breaking (grey with a $2-\mathrm{eV}$ threshold, red with a $2.5-\mathrm{eV}$ threshold, and green with a $3-\mathrm{eV}$ threshold. The shock wave probability lines correspond to the estimates done using Eqs. (4-5). The stars indicate the probabilities calculated using Eq. (5) for the direct counts obtained for four values of LET (only for two of these values the probability is above $10^{-3}$; for the highest value of LET, all probabilities are close to unity and stars overlap).

$$
\rho_{\text {max }}=\rho_{0} \sqrt{\frac{\mathrm{LET}_{\text {crit }}}{\mathrm{LET}_{\text {cri }}}},
$$

where $\mathrm{LET}_{\text {crit }}=5 \mathrm{keV} / \mathrm{nm}$ is the smallest value of LET at which the shock wave effect starts dominating over chemical effects at the distance of $\rho_{0}=2 \mathrm{~nm}$.

The above probability for chemical effects is proportional to the fluence of secondary particles. According to the predictions of Ref. 19 , in the region of interest, this quantity is inversely proportional to the distance from the ion's path. On the other side, the number of breaks $N$ is proportional to the pressure on the wave front, and, therefore, inversely proportional to the distance squared. The comparison of these effects, shown in Fig. 3 at a given radius, can be extended to different radii. In Fig. 4 , the ratio of probabilities, $P_{c h} / P_{s w}$, is shown as a function of the distance $\rho$ from the path and the LET. Yellow and red areas indicate the dominance of direct damage by shock waves. These predictions are done using $E_{0}=2 \mathrm{eV}$. Even this threshold value may be overestimated, for instance, in the presence of solvated electrons attached to a DNA molecule, it may be several times smaller ${ }^{18}$.

Now we want to turn our discussion to another effect, also related to the shock wave, which is playing a significant role at values of LET that are large enough to produce a shock wave, but not sufficiently large to cause the covalent bond rupture. The analysis shows that even at small values of LET, typical for the plateau region in the LET dependence on depth (well before the Bragg peak), the shock wave is formed, however it damps and becomes acoustic at radii under $10 \mathrm{~nm}$; at LET $=0.9 \mathrm{keV} / \mathrm{nm}$ shock waves propagate further than $10 \mathrm{~nm}$.

The study done in Ref. 9 suggests that there is a considerable collective radial flow, which propagates the high density region of medium with the wave front. The maximal mass flux density of a cylindrical shock wave is given by $\varrho_{f} u$, where $\varrho_{f}$ is the maximum density of water, which occurs at the wave front, and $u$ is the radial velocity. This flux density is inversely proportional to radius $\rho$ and is linear with respect to the $\sqrt{\text { LET }}$ (see the supplemental material). It sharply drops to zero in the wake of the wave along with the density. A sharp rarefication of the volume in the wake of the wave follows from the results of Ref. 9. This is the effect of cavitation on a nanometre scale and due to this effect the water molecules of the hot cylinder along with all reactive species formed in this cylinder are pushed out by the radial flow. Such a mechanism of propagation of reactive species is competitive with the diffusion mechanism, studied in Monte Carlo simulations done using track structure $\operatorname{codes}^{20}$.

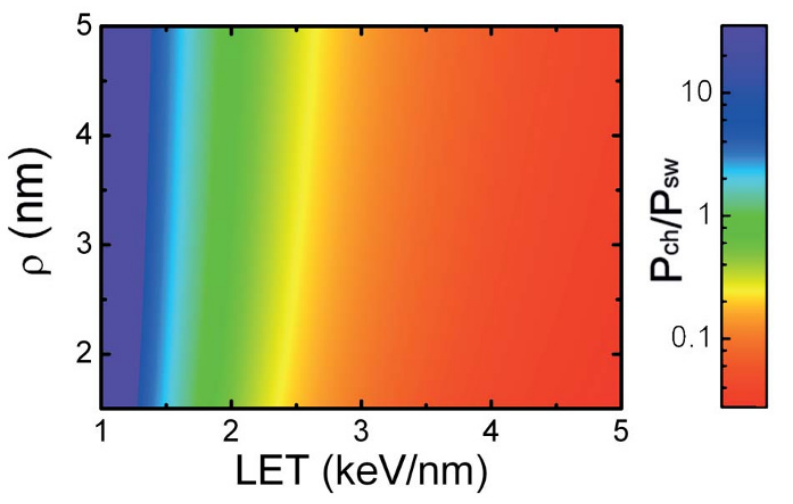

Figure $4 \mid$ The ratio of the probabilities, $P_{c h} / P_{s w}$, for producing at least one SSB in a 3-base-pair segment of DNA molecule. Probability $P_{s w}$ is calculated using Eqs. (4-5) with $E_{0}=2 \mathrm{eV}$. Yellow and red areas indicate the dominance of direct damage by shock waves. 
Intriguingly, the cylindrical shock wave accomplishes the transfer of reacting species such as hydroxyl and solvated electrons, which play important roles in chemical DNA damage ${ }^{18,21}$ much more effectively than the diffusion mechanism. Indeed, the time at which thewave front reaches radius $\rho$ is given by $1.35 \rho^{2} \sqrt{\varrho / \mathrm{LET}}$, where $\varrho=1 \mathrm{~g} / \mathrm{cm}^{3}$ is the density of undisturbed water. This time has to be compared to diffusion times, which can be estimated for different reacting species as $\rho^{2} / D$, where $\rho$ is the distance from the ion's path and $D$ is the corresponding diffusion coefficient. The ratio of these times is equal to $1.35 \sqrt{\varrho / \text { LET } D}$. For all relevant species, the diffusion coefficient is less than $10^{-4} \mathrm{~cm}^{2} / \mathrm{s}^{22}$. Therefore, the above ratio is less than $10^{-3} / \sqrt{\mathrm{LET}(\mathrm{keV} / \mathrm{nm})}$, which is much less than unity even for protons. For instance, for carbon ion projectiles, the wave front reaches $5 \mathrm{~nm}$ from the path in $2.8 \mathrm{ps}$ after the ion's traverse, while hydroxyl radicals reach the same distance via the diffusion mechanism in about $9 \mathrm{~ns}$, a more than 3000 times longer time.

In conclusion, the effects following from the local heating of the medium in the vicinity of ion paths are quite striking. Indeed, our MD simulations of a shock wave on a nanometre scale, initiated by an ion propagating in tissue-like medium, demonstrate that such a wave generates stresses, capable of breaking covalent bonds in a backbone of a DNA molecule located within $1.5 \mathrm{~nm}$ from the ion's path when LET exceeds $4 \mathrm{keV} / \mathrm{nm}$ and this becomes the dominating effect of strand breaking at LET $\gtrsim 5 \mathrm{keV}$. [These values correspond to our conservative estimates $\left(E_{0}=3 \mathrm{eV}\right)$. They may be much lower if the actual thresholds appear to be smaller $^{18}$.] The LET of $\sim 4-5 \mathrm{keV} / \mathrm{nm}$ corresponds to the Bragg peak values for ions close to $\mathrm{Ar}$ and heavier in liquid water. Besides the dramatic effects at such high values of LET, we found that weaker shock waves produced by carbon ions or even protons transport the highly reactive species, hostile to DNA molecules, much more effectively than diffusion. This effect can become the key for the experimental observation of thermomechanical effects. The acoustic waves induced by ions were detected ${ }^{23,24}$, and now we are looking forward to the experimental validation of the shock waves and their effects.

The notion of thermomechanical effects represents a paradigm shift in our understanding of radiation damage due to ions and requires re-evaluation of relative biological effectiveness because of collective transport effects for all ions and direct covalent bond breaking by shock waves for ions heavier than argon. These effects will also have to be considered for high-density ion beams, irradiation with intensive laser fields, and other conditions prone to causing high gradients of temperature and pressure on a nanometre scale.

\section{Methods}

The crystallographic structure of a nucleosome for MD simulations was taken from the Protein Data Bank (PDB) using the PDB ID 3LEL ${ }^{25}$. The nucleosome was surrounded by a water cylinder of radius of $17 \mathrm{~nm}$ and length of $7.2 \mathrm{~nm}$. Sodium and chlorine ions were added to the solution and the whole system was electrically neutral. A nucleosome has a roughly disk-like shape and the ion's path was chosen to be parallel to the axis of the disk. Periodic boundary conditions were applied along the $z$ axis of the water cylinder. The repulsive boundary condition was applied on the cylinder's $x y$ plane during equilibration of the system. After the optimisation of the system using a conjugate gradient algorithm the system was equilibrated for 50 ps at $310 \mathrm{~K}$ using Langevin thermostat with a damping constant equal to $5 \mathrm{ps}^{-1}$. All the MD simulations presented in this work were conducted using NAMD software package $^{15}$ and the CHARMM $22^{16}$ force field. Water molecules were simulated using the TIP3 model $^{16}$. For more detail of these simulations please see the supplemental material.

For each value of LET we have conducted five independent simulations in order to acquire higher statistics and have recorded the potential energy in each bond every fs of the simulations. Due to the vibrations of covalent bonds, the potential energy oscillates with a period of $\sim 10 \mathrm{fs}$ (half of a period of covalent bond vibrations). We assume that the event of bond breakage may occur only when the bond is under stress, i.e., in the vicinity of the moments at which the dependence of potential energy on time reaches a local maximum. The energies (for the selected bonds) at all such moments within a chosen period of time are recorded in our simulations. The total number of energy records $N_{r}$ corresponding to potential breaks of the chosen covalent bonds within a given time can be approximately estimated by twice the number of vibration periods of the covalently bonded atoms within this time multiplied by the number of bonds.

1. Surdutovich, E. \& Solov'yov, A. Multiscale physics of ion-beam cancer therapy. J. Phys.: Conf. Ser. 373, 012001 (2012).

2. Baccarelli, I., Gianturco, F., Scifoni, E., Solov'yov, A. \& Surdutovich, E. Molecular level assessments of radiation biodamage. Eur. Phys. J. D 60, 1 (2010).

3. Schardt, D., Elsässer, T. \& Schulz-Ertner, D. Heavy-ion tumor therapy: Physical and radiobiological benefits. Rev. Mod. Phys. 82, 383-425 (2010).

4. Solov'yov, A., Surdutovich, E., Scifoni, E., Mishustin, I. \& Greiner, W. Physics of ion beam cancer therapy: A multiscale approach. Phys. Rev. E79, 011909 (2009).

5. Cost nano-ibct - nanoscale insights into ion beam cancer therapy. URL http:// www.cost.eu/domains_actions/mpns/Actions/nano-ibct/. Accessed on 01/2013.

6. Nano-ibct: Nanoscale insights into ion beam cancer therapy (nano-ibct). URL http://fias.uni-frankfurt.de/nano-ibct/. Accessed on 01/2013.

7. Surdutovich, E. et al. Ion-induced electron production in tissue-like media and DNA damage mechanisms. Eur. Phys. J. D 51, 63-71 (2009).

8. Toulemonde, M., Surdutovich, E. \& Solov'yov, A. Temperature and pressure spikes in ion-beam cancer therapy. Phys. Rev. E 80, 031913 (2009).

9. Surdutovich, E. \& Solov'yov, A. Shock wave initiated by an ion passing through liquid water. Phys. Rev. E 82, 051915 (2010).

10. Obolensky, O. et al. Ion beam cancer therapy: Fundamental aspects of the problem. Nucl. Inst. Meth. B 266, 1623-1628 (2008).

11. Schiwietz, G. \& Grande, P. L. Improved charge-state formulas. Nucl. Instr. Meth. B 175-177, 125-131 (2001).

12. Abril, I., Garcia-Molina, R., Denton, C., Kyriakou, I. \& Emfietzoglou, D. Energy loss of hydrogen- and helium-ion beams in dna: Calculations based on a realistic energy-loss function of the target. Radiat. Res. 175, 247-255 (2011).

13. Surdutovich, E. \& Solov'yov, A. V. Double strand breaks in dna resulting from double ionisation events. Eur. Phys. J. D 66, 206 (2012).

14. Solov'yov, I. A., Yakubovich, A. V., Nikolaev, P. V., Volkovets, I. \& Solov'yov, A. V. Meso bio nano explorer - a universal program for multiscale computer simulations of complex molecular structure and dynamics. J. Comp. Chem. 33, 2412-2439 (2012)

15. Phillips, J. C., Braun, R., Wang, W. et al. Scalable molecular dynamics with namd. J. Comp. Chem. 26, 1781-1802 (2005)

16. MacKerell, A. et al. All-atom empirical potential for molecular modeling and dynamics studies of proteins. J. Phys. Chem. B 102, 3586-3616 (1998).

17. Range, K., McGrath, M. J., Lopez, X. \& York, D. M. The structure and stability of biological metaphosphate, phosphate, and phosphorane compounds in the gas phase and in solution. J. Am. Chem. Soc. 126, 1654-1665 (2004).

18. Smyth, M. \& Kohanoff, J. Excess electron interactions with solvated dna nucleotides: Strand breaks possible at room temperature. J. Am. Chem. Soc. 134, 9122-9125 (2012).

19. Surdutovich, E. \& Solov'yov, A. V. Random walk approximation for the radial dose dependence. Eur. Phys. J. D 66, 245 (2012).

20. Nikjoo, H., Uehara, S., Emfietzoglou, D. \& Cucinotta, F. A. Track-structure codes in radiation research. Radiat. Meas. 41, 1052 (2006).

21. Sanche, L. Low-energy electron interaction with dna: Bond dissociation and formation of transient anions, radicals, and radical anions. In Greenberg, M. (ed.) Radical and radical ion reactivity in nucleic acid chemistry, 239 (J. Wiley \& Sons, Inc., New York, 2010).

22. LaVerne, J. Radical and molecular yields in the radiolysis of water with carbon ions. Radiat. Phys. Chem. 34, 135-143 (1989).

23. Sun, Y. \& Nath, R. Pressure wave generated by the passage of a heavy charged particle in water. Med. Phys. 20, 633 (1993).

24. Baily, N. A. A review of the processes by which ultrasound is generated through the interaction of ionizing radiation and irradiated materials: Some possible applications. Med. Phys. 19, 525-532 (1992).

25. Wu, B., Mohideen, K., Vasudevan, D. \& Davey, C. A. Structural insight into the sequence dependence of nucleosome positioning. Structure 18, 528 (2010).

\section{Acknowledgements}

We are grateful to Walter Greiner for fruitful discussions, Bradley Roth who critically read the manuscript, and the support of European COST Action MP1002 "Nano-scale insights in ion beam cancer therapy." A.V.Y. thanks Stiftung Polytechnische Gesellschaft Frankfurt am Main for financial support and acknowledges the Center for Scientific Computing (CSC) of the Goethe University Frankfurt for providing with computer facilities.

\section{Author contributions}

The concept, idea and the text were mainly designed by E.S. and A.V.S., while the M.D. simulations and figures were carried out and prepared by A.V.Y. The analysis of the results of the simulations were performed by all three authors.

\section{Additional information}

Supplementary information accompanies this paper at http://www.nature.com/ scientificreports 
Competing financial interests: The authors declare no competing financial interests. License: This work is licensed under a Creative Commons

Attribution-NonCommercial-NoDerivs 3.0 Unported License. To view a copy of this license, visit http://creativecommons.org/licenses/by-nc-nd/3.0/
How to cite this article: Surdutovich, E., Yakubovich, A.V. \& Solov'yov, A.V. Biodamage via shock waves initiated by irradiation with ions. Sci. Rep. 3, 1289; DOI:10.1038/srep01289 (2013). 